ISBN 978-81-933894-1-6

5th International Conference on Future Computational Technologies

(ICFCT'2017)

Kyoto (Japan) April 18-19, 2017

\title{
Application of Mb/M/1 Bulk Arrival Queueing System to Evaluate Key-In System in Islamic University of Indonesia
}

\author{
Novaldy Pratama Putra ${ }^{1}$, Ravanel Yosandi Lail ${ }^{1}$ and Atina Ahdika ${ }^{2}$ \\ ${ }^{1}$ Student, Department of Statistics in Islamic University of Indonesia \\ ${ }^{2}$ Lecturer, Department of Statistics in Islamic University of Indonesia
}

\begin{abstract}
According to queueing theory, customers arrive randomly follow to a Poisson process. In many practical queueing, customers come in batches. This situation can be modeled as queues process with bulk arrivals. The analysis of this paper help the decision maker decide to control arrival rates and service rates to reduce the possibility of key in system would be blocked. This paper also simulated in detail the correlation between performance measurement and traffic intensity for various bulk size
\end{abstract}

Keywords: Bulk Arrival, Key in, Stochastic Process, System Evaluation

\section{Introduction}

In any kind of service can not be separted with the queue. It cause by the needed foe the service is bigger than the capacity of service. This conditon often seen in daily life, like queue to get the cinema ticket, queue to get the doctor receipt, queue in minimarket, etc. The queue is a process that associate by the arrival of customer and the limited capacity by the service. It makes the customer waiting in the line until they in service system then leaving the system.

Crescenzo et all [1] study of a birth-death process $\mathrm{N}(\mathrm{t})$ with a reflecting state at 0 we propose a method able to construct a new birth-death process $\mathrm{M}(\mathrm{t})$ defined on the same state-space. Under a suitable assumption we obtain the conditional probabilities, the mean of the process, and the Laplace transforms of the downward firstpassage-time densities of $\mathrm{M}(\mathrm{t})$.

Ghimire [2] study of $\mathrm{M} / \mathrm{M} / 1$ queue with heterogeneous arrival and departure with the provision of server vacations and breakdowns. Customer arrive service facilities with poison process and exponential service time distribution. They find the mean queue length, mean waiting time in queue and system, average number of customers in the system. They use generating function method to find these measures of performance. The numerical results are obtained to cite the applicability of model in the real life situations.

Maurya [3] study the bulk arrival retrial queueing MX / G1, G2, / 1 model with two phase service and Bernoulli vacation schedule wherein first phase service is essential and the next second phase service is optional. The objective of this paper is to investigate the steady state behavior of the bulk arrival retrial queueing MX / G1, G2, / 1 model with two phase service and Bernoulli vacation schedule. By introducing supplementary variables, Chapman Kolmogorov equations are established and then the probability generating functions (PGFs) for first phase essential service (FPES), next phase optional service (NPOS) and working vacation and for the number of the customers in the orbit at an arbitrary epoch are investigated successfully.

In this paper, we apply bulk arrival to solve the problem in each semester in Islamic University of Indonesia (UII). One of the biggest problems of each semesters is Plan of Study Card or we call KRS. KRS is proof of an active student in the semester. It has function as Card Exam Participants. KRS must be taken every time student 
take the exam. KRS at UII better known as key-in. UII students did key-in according to the schedule set by the department campus. Key in can be acces online by $\log$ in to the website unisys.uii.ac.id. UII set policies simultaneously for each department. Campus policy establishes that students can choose either courses, schedules, until the lecturer. As a result of the use of the server simultaneously not all students can be directly entered into the system as it relates to the speed of each network and server capacity of the students themselves. When this happens, the student can not choose courses and lecturers desired so the schedule that have been prepared previously will be very different to that obtained.

Based on the problems that appear each semester, it is necessary to do research related to the number of students in the system and how long it takes to complete the process of key-ins This situation can be modeled as queues with bulk arrivals. This analysis can help the decision maker to determine the best system in the student key-in process in islamic University of Indonesia using the model.

\section{Queueing Theori}

Queueing is a process that deals with the arrival of customer in almost any kind of service. The service begins when the queue of custumers who need the sevice arrive to service facility, waiting in the queue line service. The customers come rom a population that is called the input source.

According to Hillier and Lieberman [4] said that the queueing process is a process associated with the arrival of a customer at queue system, than waiting in the line service until server choose customer based on service dicipline and get service then leave the system after being served.

Queueing system is association of customer, waiters, and some regulation that organize the arrival of customer and the service. Queueing is a birth death process with the population that consist the customer in line for waiting or in service.

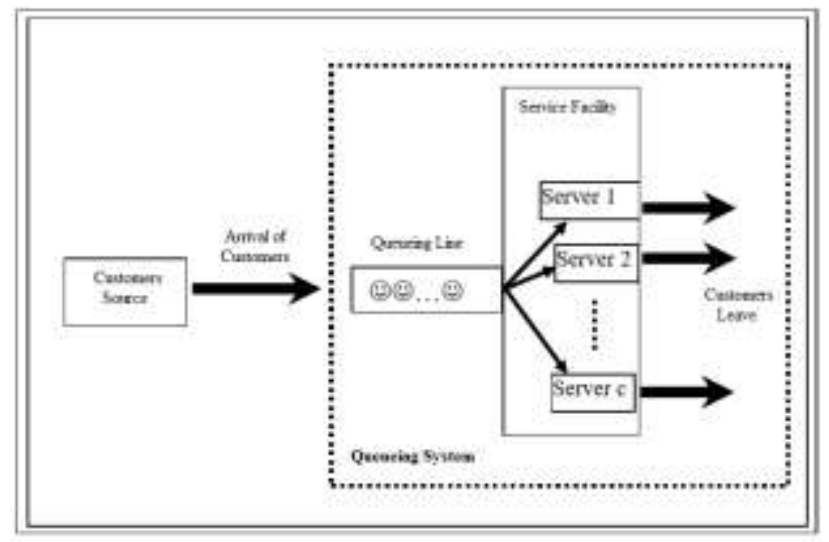

Fig. 1: Diagram of Queue

\section{Birth Death Process}

Queueing system is association of customer, waiters, and some regulation that organize the arrival of customer and the service. Queueing is a birth death process with the population that consist the customer in line for waiting or in service.

Markov Chain analysis is one of the statistical methods which can be used to estimate the future in probabilistic point of view. The analysis works by using information from the past to obtain the information of the future.

CTMC can be described as a birth and death process, where the process which have states $\{0,1,2, \ldots\}$ can move only from state $i$ to state $i+1$ or to state $i-1$. A birth-death (BD process) process refers to a Markov process with a discrete state space. The statst of which can be enumerated with index $i=0,1,2, \ldots$ such that state transitions can occur only between neighbouring states, $i$ to $i+1$ or $i-1$. The relationship between the birth death rate and state transition probabilities are:

The relationship among the states is showed by Figure 2 . 


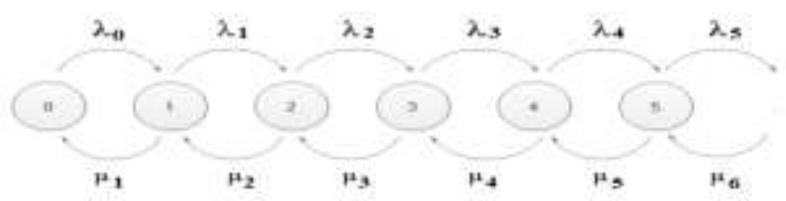

Fig. 2 Diagram of Birth Death Process

Where the transition rates are

$$
q_{i, j}=\left\{\begin{array}{ccc}
\lambda_{i} & \text { if } & j=i+1 \\
\mu_{i} & \text { if } & j=i-1 \\
0 & \text { others } &
\end{array}\right.
$$

The transition rate $q_{i, j}$ can be written in matrix generator $Q$ form as follow

$$
Q=\left(\begin{array}{ccccc}
q_{0,0} & q_{0,1} & q_{0,2} & \cdots & q_{0, n} \\
q_{1,0} & q_{1,1} & q_{1,2} & \cdots & q_{1, n} \\
q_{2,0} & q_{2,1} & q_{2,2} & \cdots & q_{2, n} \\
\vdots & & & \ddots & \vdots \\
q_{n, 0} & q_{n, 1} & q_{n, 2} & \cdots & q_{n, n}
\end{array}\right)=\left(\begin{array}{ccccc}
-\lambda_{0} & \lambda_{0} & 0 & \cdots & 0 \\
\mu_{1} & -\left(\lambda_{1}+\mu_{1}\right) & \lambda_{1} & \cdots & 0 \\
0 & \mu_{2} & -\left(\lambda_{2}+\mu_{2}\right) & \cdots & 0 \\
\vdots & & & \ddots & \vdots \\
0 & 0 & 0 & &
\end{array}\right)
$$

From matrix $Q$, we can obtain time-dependent solution of probability in state $n$ by solving the global balance condition

Where $\pi(t)=\left[\begin{array}{llll}\pi_{0}(t) & \pi_{1}(t) & \pi_{2}(t) & \cdots\end{array}\right]$

$$
\frac{d}{d t} \pi(t)=\pi(t) \cdot Q
$$

\section{Bulk Arrival}

In this queuing system, a customers arrive in groups with the group size is $X$, where in general $X$ is variable positive random. In this discussion, the customer arrive by distribution poisson with arrival rate $\lambda$, and there is a server have exponential distribution service time with the pace of service $\mu$, Where customers are served individually with FIFO (First In First Out) queuing discipline. Design services in this queue system is the Single Channel Single Phase. Notation for single server queuing model with the group arrival (bulk arrival) is $M^{X} / M / 1$.

Examples of situations in a queuing system where customers arrive the arrival of a group of customers in a group in a restaurants and letters that came in the post office. illustration queuing system with the groups arrival (bulk arrival) shown in figure 3.

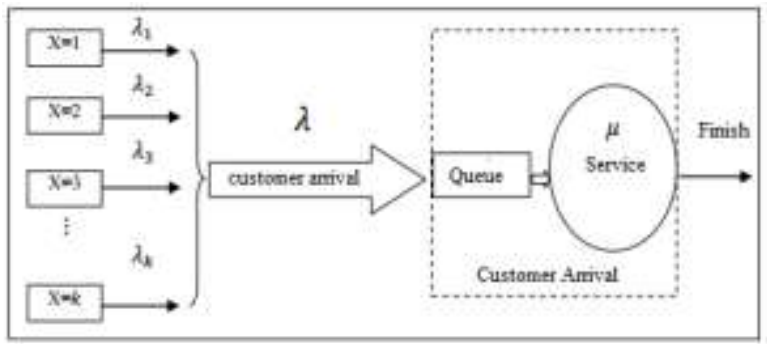

Fig. 3: Queue System $M^{X} / M / 1$

If $\mathrm{X}$ is a random variable states the size of the group with the function of probability ak $=\mathrm{P}(\mathrm{X}=\mathrm{k})$ with $\mathrm{k} \geq$ 1 based on (Purcell \& Varberg, 1987:49) if $\mathrm{S}(\mathrm{x})$ is the number of polinomial series on hose $\mathrm{I}=\{\mathrm{x} \mid-1<\mathrm{x}<1\}$ so that $\mathrm{S}(\mathrm{x})=\sum_{n=1}^{\infty} x^{n}$ The first of derivative of $\mathrm{S}(\mathrm{x})$ is $\sum_{n=0}^{\infty} n x^{n-1}$ It means definitions probability generating function (PGF) on is

The first of derivative

$$
\mathrm{A}(z)=\sum_{k=1}^{\infty} a_{k} z^{k},|z| \leq 1
$$




$$
A^{\prime}(z)=\sum_{k=1}^{\infty} k a_{k} z^{k-1}
$$

$$
A^{\prime}(1)=\sum_{k=1}^{\infty} k a_{k}
$$

Based on Bain \& Engelhardt[5] if $\mathrm{X}$ is a discrete random variable with the function of probability $\mathrm{f}(\mathrm{x})$ then the expected value of $\mathrm{X}$ is defined as $\mathrm{E}(\mathrm{X})=\Sigma_{x} x f(x)$. The equation above is the expectation value of $\mathrm{X}$ represented by

$$
E(X)=\sum_{k=1}^{\infty} k a_{k}=A^{\prime}(1)=\bar{a}
$$

A bulk queue is a queue model where the customer arrived and served in a groups of random size. The arrival process in bulk queue following poisson distribution withthe number of arrival per time is $\lambda$ and size of arrival is $\bar{a}$ so that the number of arrival per time in queue system is $\lambda \bar{a}$.

The steady state transition diagram for this model is shown in figure 4.

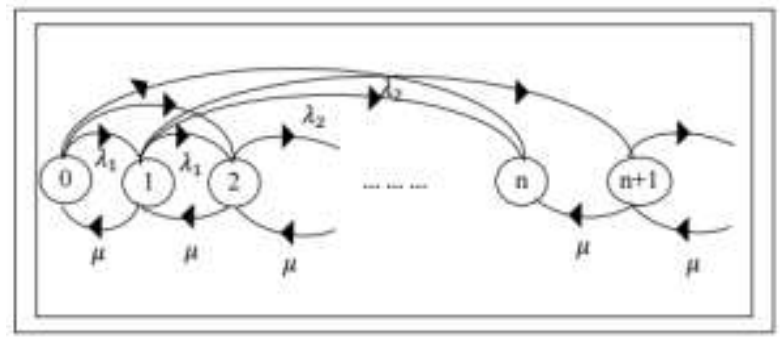

Fig. 4: Transition diagram for general states

From generating function so we can define $G(z)$

$$
G(z)=\frac{\mu p_{0}}{\mu-\lambda \sum_{j=1}^{b} z^{j}}
$$

Since $\rho=\frac{\lambda b}{\mu}$ is the utilization of the server, we have

Therefore (5) becomes $G(z)=\frac{\mu(1-p)}{\mu-\lambda \Sigma_{j=1}^{b} z^{j}}$

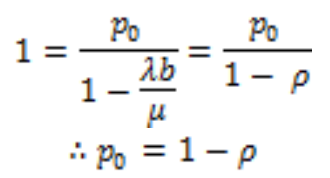

$L_{g}=\frac{\rho(1+b)}{2(1-p)}$

$W_{s}=\frac{1+b}{2 \mu(1-p)}$

$L_{q}=L_{g}-\rho$

$W_{q}=W_{s}-\frac{1}{\mu}$

\section{Result and Discussion}

The study population were taken in this research is the key-in history of all the statistics students (class of 2013). Data collection was carried out from the major of Statistics(class of 2013) at science department of UII. To retrieve the Data, one that has to do is by asking for a print captured of key-in history of each student statistics class of 2013. To obtaine key-in itself, when a student wants to enter a new semester and that's where the campus held a key-ins. Key in was conducted on 19 Februari 2016 until February 25, 2016. In the processing of key-in, the students can choose the course they want to take and can select the desired lecturers. When the students are finished, it can be seen at the key-in history. The data that were taken in the key-in history is a login process and log-out process that students performed only on February 19, 2016. The steps is as follows: 
Student opens unisys.uii.ac.id website, the following display appears in the picture below, enter the user id and password in the fields provided. the User id and password of each students are different. Once inserted, the following display that appears, and the next step is by clicking key-in ras and key-in, the following looks:

From the value of the existing transition, obtained the value $\lambda=1 / 85$ and $\mu=1 / 7004$. For the bulk value worth 5 due in 1 second can enter up to three people, so that the transition can be seen recently when four people, so that 5 can be said to be ideal. Of the value will be obtained the probability of no one in the system or the value $\mathrm{P} 0$.

$P_{0}=1-\rho=1-\frac{\lambda \mathrm{b}}{\mu}=1-\frac{\frac{1}{85} \times 5}{\frac{1}{7004}}=1-412,0803=-411.08$

From the equation above shows that $\rho>1$. This shows that the system is not effective, so then obtained $P_{0}$ minus value. After obtained $P_{0}$ value, then the value of the performance measure can be searched as follows:

$L_{s}=\frac{\rho(1+b)}{2(1-\rho)}=\frac{412,0803(1+5)}{2(1-412,0803)}=-3.0073$

$W_{S}=\frac{1+b}{2 \mu(1-\rho)}=\frac{1+5}{2 * 1 / 7004(1-412,0803)}=-51.1140969$

$L_{\mathrm{q}}=L_{g}-\rho=-3.0073-412,0803=-415.088$

$W_{q}=W_{S}-\frac{1}{\mu}=-51.1140969-\frac{1}{\frac{1}{7004}}=-7005.11$

From the above calculation values that obtained throughout the value performance of measure entirely negative because of the value of $\rho$ is very large. It does not make sense because the performance measure stating the average number of people and length of time spent in the system and in the queue.

It is necessary for simulation of how the comparison of the performance of measurement using the value $\lambda=$ $1 / 85$ and $\mu=1 / 7004$. The following results:

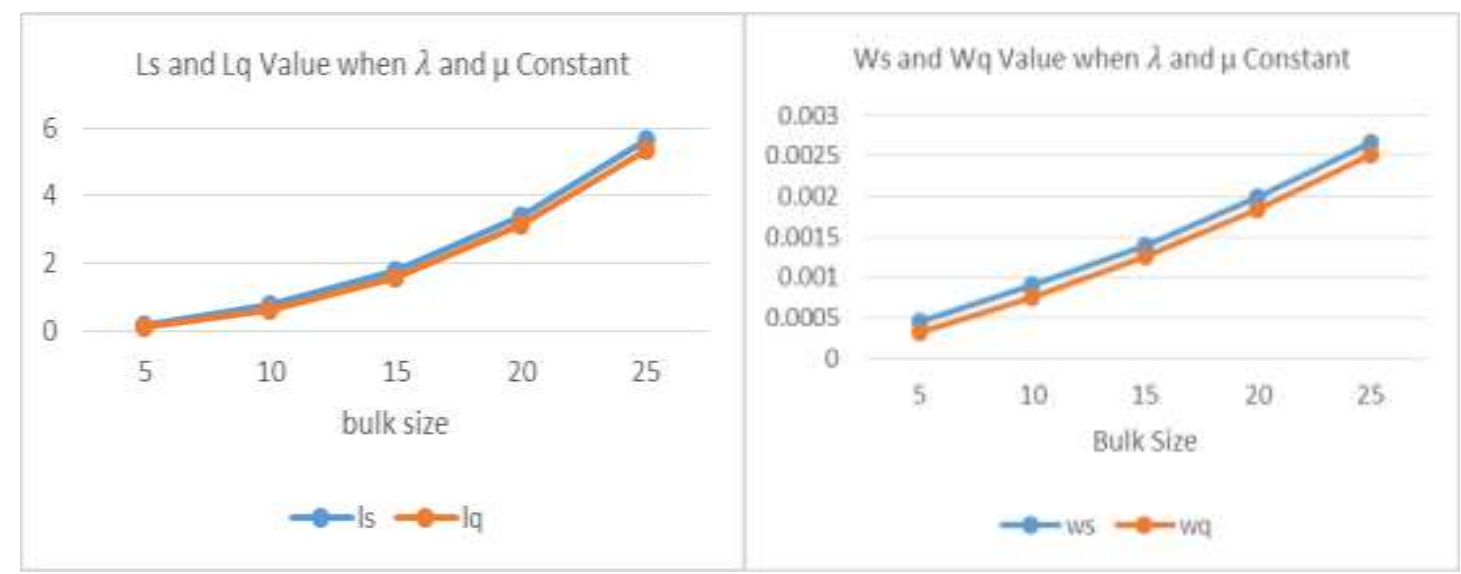

Fig. 5: Comparison Performance Meusrement when $\lambda$ and $\mu$ Constant

From the graph above it can be seen that the greater the value the greater bulk, the average number of people in the system (Ls) and in queue (Lq) will be greater. This is because more and more people are entering the system or queue together while the server is one that would make too many people either in the system or queue. Neither is the case with the time each person spends in the system (Ls) or a queue (Lq) will be even greater if the number of people in the group the greater because the server will serve one-on-one 


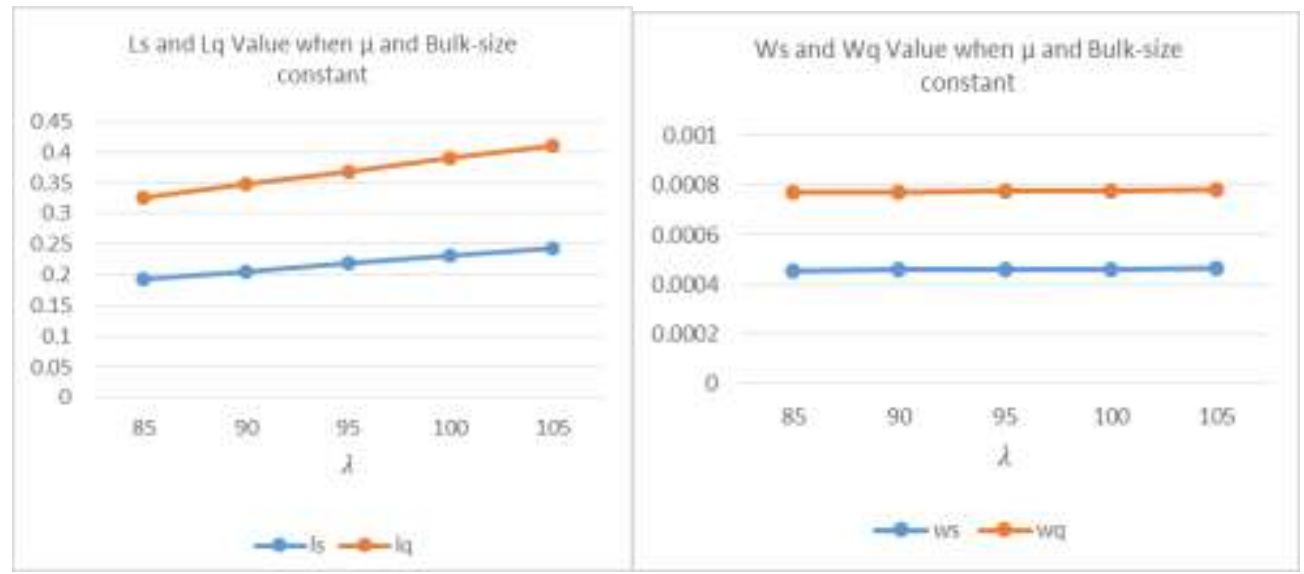

Fig. 6: Performance Measurement when $\mu$ and Bulk-size Constant

From the graph above can be seen as another measure value remains greater the value of lambda or greater then the arrival rate will cause the value of his performance measure is greater. Differences graph Ls and Lq and between Ws and Wq far enough due to changes in the value of $\lambda$ where the effects are significant for multiplication with other elements.

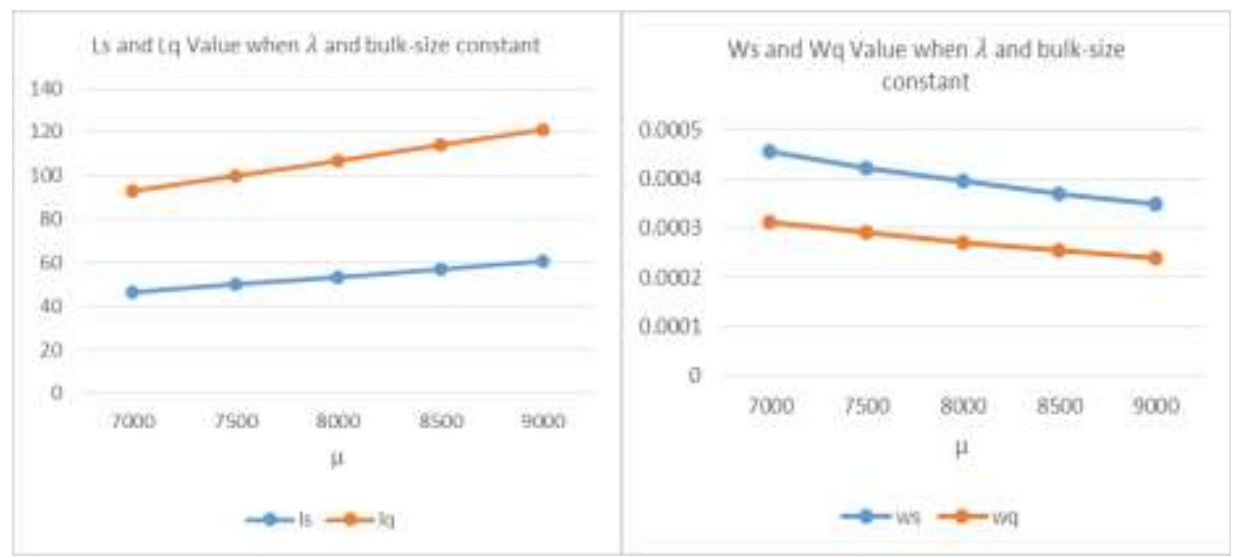

Fig. 7: Comparison Performance Measurement when $\lambda$ and Bulk-size Constant

From the chart above can be seen when the value of other fixed size, the greater the value of service rate $\mu$ or it will cause a lot of people in the system and the queue grows. But even Ls and Lq is a big plus, increasing the value of $\mu$ it will cause the value of Ws and Wq shrinking. This is because the greater the rate of service, it will cause the server power to serve customers better, which of course will lead to a queue in the queue is getting smaller

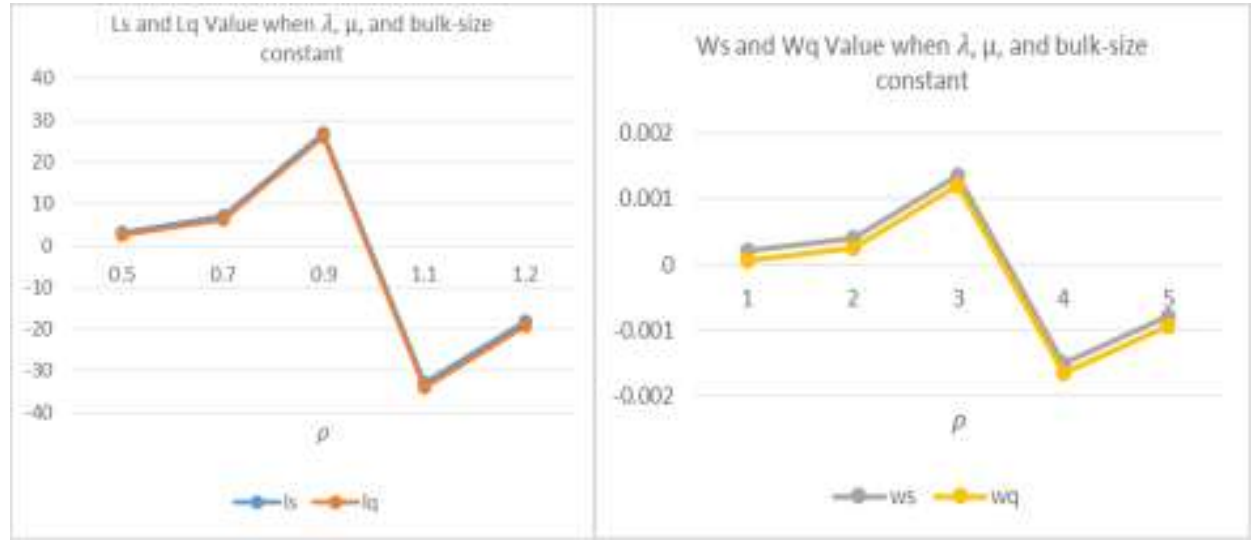

Fig. 8: Comparison Performance Measurement when $\lambda, \mu$, and Constant 
From the graph above it can be seen how the changes Ls and Lq when $\mu, \lambda$, and $b$ fixed. The greater the value of rho $(\rho)$ or traffic intensity, the greater the people and time spent in the system or queue. But it will be different when the value of $\rho$ is greater than 1, it appears that the value is negative. The increase in the number 1.2 in the equation because there is a formula $(1-\rho)$ so that these increases are only temporarily in the range $1<\rho<2$.

\section{Conclusion}

From the results that obtained it can be concluded that $\rho$ obtained greater than 1 , it means that traffic transity on key system-in RAS in UII is ineffective. The relationship between $\mu, \lambda$, and $b$ the value of Ls and Lq has a positive relationship, i.e. the greater the value of $\mu, \lambda$, and $b$, the greater the Ls and Lq. Similarly, the relationship between $\lambda$ and $b$ towards $\mathrm{Ws}$ and $\mathrm{Wq}$ also has a positive relationship, whereas the relationship between $\mu \mathrm{Ws}$ and Wq have a negative relationship.

\section{Acknowledgements}

Academic Development Directorate of Islamic University of Indonesia (BPA UII) and Directorate of Talents, Interests, and Student Welfare for funding the conference

\section{References}

[1] Crescenzo et all., A Multispecies Birth Death Immigration Process and its Diffusion Approximation, Journal of Mathematical Analysis and Applications, 442, pp. 291-316, 2016

https://doi.org/10.1016/j.jmaa.2016.04.059.

[2] Maurya V. N. (2013): Sensitivity Analysis on Significant Performance Measures of Bulk Arrival Retrial Queueing MX/(G1,G2)/1 Model with Second Phase Optional Service and Bernoulli Vacation Schedule. International Open Journal of Operations Research, 1, $01-15$.

[3] Ghimire R. P. and Ghimire S. (2011): Heterogeneous Arrival and Departure M/M/1 Queue with Vacation and Service Breakdown. Management Science and Engineering

[4] Hillier, Frederick. S dan Lieberman, Gerald. I. 1980. Introduction to. Operations Research. Holden Day, Inc. San Francisco

[5] Bain, Lee J. dan Engelhardt, Max. 1992. Introduction to Probability and. Mathematical Statistics. Duxbury Press, California 